\title{
Electron Cyclotron Emission (ECE) and Correlation ECE diagnostics on TCV
}

\author{
Matteo Fontana ${ }^{1, a}$, Laurie Porte ${ }^{1}$, and Philippe Marmillod ${ }^{1}$ \\ ${ }^{1}$ Swiss Plasma Center - EPFL - Station 13, Lausanne, $\mathrm{CH}$
}

\begin{abstract}
The Electron Cyclotron Emission (ECE) and correlation ECE diagnostics in TCV have been upgraded during its 2014 shutdown; this paper will provide a brief overview on their updated architecture and capabilities. The ECE system is equipped with two radiometers, each with 24 channels ( $750 \mathrm{MHz}$ bandwidth) looking at the plasma both from the low and high field side covering the whole vessel. Of particular interest is the possibility of acquiring signals through a vertical line of sight and a dual-axis steerable antenna to study the electron distribution function (EDF) in plasmas with Electron Cyclotron Current Drive (ECCD) or EC Resonant Heating (ECRH). The correlation ECE system has been equipped with a new independent front end connected to the steerable antenna. The main characteristics of the radiometer are the six YIG independently tunable (between 6-18 GHz) $170 \mathrm{MHz}$ bandwidth channels that can be moved on the whole LFS of the vessel. Using correlation analysis techniques it is possible to study very small temperature fluctuations. A brief presentation of some applications where these capabilities have been exploited in past TCV experiments for the study of micro instabilities characteristics is also included.
\end{abstract}

\section{Introduction}

Tokamak à Configuration Variable (TCV) is a medium sized machine for the study of the physics of magnetically confined plasmas for nuclear fusion applications. Its most relevant characteristics are the ability to work with a large range of different plasma shapes, its versatile heating system and the large variety of diagnostic systems available for the study of the plasma. In the past year the Electron Cyclotron Emission (ECE) diagnostic systems have been significantly upgraded. This kind of diagnostic allows the measurement of local electron temperature with high spatial and temporal resolution.

\section{ECE}

TCV has been equipped for the first time with ECE diagnostics in 2002 (HFS) and 2003 (LFS). In the following section we will describe the current status of the ECE systems on TCV.

\subsection{Lines of sight}

The ECE system of TCV is composed of two separate radiometers usually connected to a set of lines of sight looking from the HFS and the LFS, perpendicular to the magnetic field. Each radiometer can be connected to one of two antennas, one on the mid plane of the tokamak $(z=0)$ and the other at $z=21 \mathrm{~cm}$. All of these transmit the signal up to the radiometers through an overmoded 1" circular waveguide ending in a rectangular section. The $z=0$

\footnotetext{
a e-mail: matteo.fontana@epfl.ch
}

LFS line of sight uses as a focusing element a polytetrafluoroethylene (PTFE) lens placed outside of the vessel port, while the one at $z=21 \mathrm{~cm}$ uses an in-vessel system composed by an elliptical mirror. Vacuum is assured by a quartz window. The HFS lines of sight are similar to the second LFS one with the difference that the waveguides run behind the tiles of the central column and bring the signal towards the upper part of the vessel where the vacuum break is provided by a $200 \mu \mathrm{m}$ Mylar foil. The antenna pattern of these lines allows the study of modes with $k_{\theta}<0.3 \mathrm{~cm}^{-1}$, except for the LFS $z=0$ one which is limited to $k_{\theta}<0.7 \mathrm{~cm}^{-1}$ at the vessel center.

\subsubsection{Alternative lines of sight}

In addition to these four lines of sight, TCV is equipped with a steerable receiver connected to the LFS and CECE radiometers, and a vertical receiver, whose signal can be transmitted to both HFS and LFS radiometers. Both lines of sight can be used to obtain information on the electron energy distribution function. The vertical line of sight consists of a focusing ellipsoidal mirror and a telescope system with a view from the top of the vessel, perpendicular to the magnetic field. A beam dump (reflectivity $<0.1 \%$ ) is placed at the bottom of the vacuum chamber. This line of sight can be considered to be looking along a surface of constant magnetic field. Neglecting the nonideal antenna pattern and Doppler shifted emissions, different frequency components of the signal can be related to electrons with different kinetic energies (relativistic broadening) [1]. This antenna is connected with a mechanical switch both to the HFS and LFS radiometers allowing the use of both sets of channels [2]. 
Another possible line of sight is a steerable antenna positioned at $z=45 \mathrm{~cm}$ (upper lateral). A detailed description of this antenna was provided by Goodman [3]. The movable mirrors allow one to rotate the line of sight with respect to a major radius $\left(\theta_{L}\right)$ and around it $\left(\phi_{L}\right)$, describing a cone trajectory. $\theta_{L}$ can be swept during a shot from 7 to $55^{\circ}$ in $500 \mathrm{~ms}$. This antenna will be shared among different diagnostics: doppler backscattering reflectometry, profile reflectometry, CECE and LFS ECE. The greatest advantages of this tool in ECE studies are the smaller spot size of the antenna (allowing the study of modes with $k_{\theta}<1.12 \mathrm{~cm}^{-1}$ ) and the possibility of using it to perform oblique measurements of the EC radiation. This is particularly interesting for plasma discharges where ECCD is present since it makes possible to study the difference between emission in co- and counter-current directions to get informations on the effects of current drive on the EDF.

\subsection{Radiometer}

The system has been designed to study second harmonic extraordinary mode (X2) electron cyclotron radition. After the circular to rectangular transition, the collected radiation passes through a couple of notch filters to block out the power from the X2 and X3 ECRH systems and then enters the two radiometers (whose scheme is presented in figure ??). The incoming radiofrequency (RF) power is divided in a directional coupler and directed to two parallel heterodyne mixing stages, each connected to a different frequency local oscillator (LO): 63.9 and $82.5 \mathrm{GHz}$ for the LFS radiometer, 76.5 and $94.5 \mathrm{GHz}$ for the HFS one. Image-reject filters are placed before the mixing stages. In both radiometers the high frequency branch of the front end includes an isolator which prevents higher frequency LO power from the mixing stage from returning back to the lower frequency branch. The downconverted signals are then transmitted into low noise amplifiers (LNA) with $40 \mathrm{~dB}$ gain over the $2-20 \mathrm{GHz}$ interval. These LNAs show $1 \mathrm{~dB}$ gain compression at $20 \mathrm{dBm}(\mathrm{P} 1 \mathrm{~dB})$ and $3^{\text {rd }}$ harmonic distortion after $30 \mathrm{dBm}$ (IP3). After amplification each signal passes through a twelve-way power divider, splitting the IF into 24 channels, each characterised by a $750 \mathrm{MHz}$ bandwidth band-pass filter. Each mixing stage is thus connected to 12 channels spaced by $1.5 \mathrm{GHz}$ in the 2-20 GHz interval. Ultimately the RF bandwidths of the HFS and LFS radiometers are respectively $78-114 \mathrm{GHz}$ and $66-100 \mathrm{GHz}$ allowing us to make measurements over almost all the radial profile of TCV as it is shown in figure 2 (a) and (b).

The HFS radiometer has the additional possibility of choosing which RF signal to send into which set of channels (shifted $750 \mathrm{MHz}$ one with respect to the other) through a couple of electronic switches, further increasing the flexibility in the choice of the resonant layers that can be analysed.

After being filtered the signals are rectified using a set of unbiased Schottky diodes (working in the 2-18 or 2-26 $\mathrm{GHz}$ interval), acquired at $200 \mathrm{kHz}$, low pass filtered (100 $\mathrm{kHz}$ ) and amplified in a remotely controlled variable gain amplifier. The response curve of the diodes is non-linear up to $-17 \mathrm{dBm}$.

\subsection{Capabilities}

The ECE system in TCV has been used in a wide range of plasma scenarios. Together, the HFS and LFS channels span the whole plasma minor radius. The $750 \mathrm{MHz}$ bandwidth of an output channel corresponds to plasma volumes with different widths as the magnetic field varies over the radial profile: in the core, one channel corresponds approximately to a $1 \mathrm{~cm}$ wide region while in the HFS or in the LFS it would respectively be smaller and larger. The poloidal spot size varies for the different lines of sight limiting the maximum values of $k_{\theta}$ that can be resolved as mentioned before.

The choice of having two radiometers looking at the vessel from two different sides was made to allow a greater flexibility in the possible applications of the system. For example the LFS system makes it possible to study the bulk plasma temperature even in conditions where ECRH or ECCD generate a consistent fraction of suprathermal electrons: ECE radiation coming from this population, having a lower frequency than that of the correspondent emission layer due to relativistic effects, would be reabsorbed before reaching the LFS receivers. Instead, the HFS system can observe radiation emitted by these fast particles, giving the possibility to compare the results between LFS and HFS data coming from similar volumes in the plasma.[4]

\section{Correlation ECE}

The correlation ECE system in TCV was installed in 2007 but only with the last upgrade did it acquire an independent front end from the LFS ECE. Its working principle is to apply spectral decorrelation to ECE signals coming from plasma volumes very close to each other but not overlapped: if the distance between the volumes is chosen correctly (typically the turbulence correlation length), small scale modes will still be correlated while the thermal noise of the two channels will not. It is hence possible to lower the minimum fluctuation detectable by the radiometer $\left(\delta T_{e} / T_{e}\right)$.[5]

\subsection{Line of sight and radiometer}

The newly upgraded CECE system in TCV uses the steerable receiver antenna. The RF stage of the CECE radiometer is similar to those, already discussed, of the standard ECE: two notch filters stop the ECRH power from reaching the mixing stage, a directional splitter divides the power between two mixers connected with LOs at 81.9 and 61.1 GHz, the lower side band is rejected and the down converted signals are amplified in LNAs with $30 \mathrm{~dB}$ gain over the $2-20 \mathrm{GHz}$ interval and $\mathrm{P} 1 \mathrm{~dB}=10 \mathrm{dBm}$. Only one of the signals coming from the two mixers is transmitted to the IF stage and that can be manually switched between shots. The IF stage consists of a six-way power splitter 


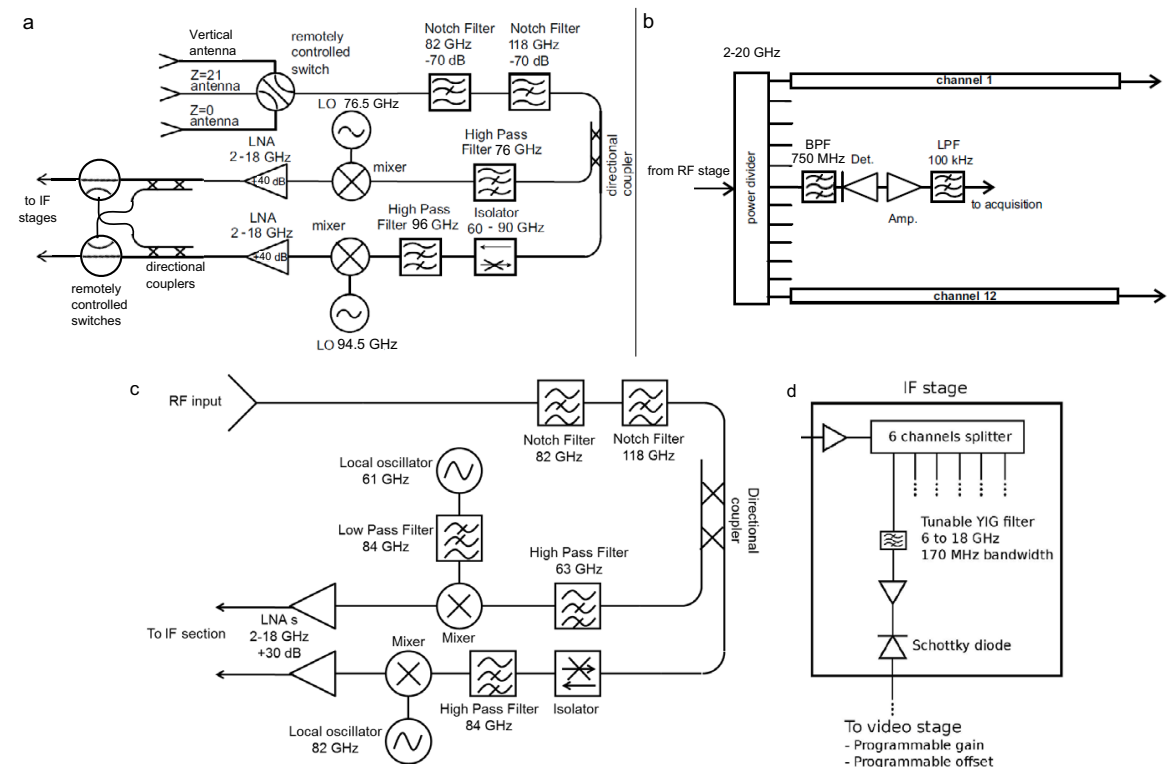

Figure 1. Schematics of the HFS (a RF stage, b IF stage) and CECE (c RF stage, d IF stage) radiometers. The LFS radiometer is identical to the HFS one except for the last section of the RF stage (the signal goes from the LNAs directly into the power dividers), the antenna connections and the working frequencies of LOs and other components.

leading to six YIG filters (170 MHz bandwidth), whose center frequency can be remotely adjusted between 6 and $18 \mathrm{GHz}$, each followed by another stage of amplification (20dB gain, $20 \mathrm{dBm}$ P1dB, $27 \mathrm{dBm}$ IP3) and an unbiased Schottky diode for rectification. The voltage signal is sampled at $1.67 \mathrm{MHz}$, low pass filtered at $450 \mathrm{kHz}$ and then goes through video cards with programmable gains and offsets. A scheme of the CECE radiometer is presented in figure 1 .

\subsection{Capabilities}

The newly upgraded CECE diagnostic is a highly versatile system: it can cover the $67-79 \mathrm{GHz}$ or $88-100 \mathrm{GHz}$ frequency intervals, depending on which mixer is being used, as shown in 2 (c), allowing to samle the whole LFS of the minor radius. Moreover the channels' frequencies, and hence the plasma volumes analysed, can be varied from one shot to another, further increasing the flexibility of the system. Having six simultaneous measurements allows to perform cross correlation analysis in up to three different plasma regions and estimate the correlation length of turbulent modes in a single shot. The radial resolution is mainly limited by relativistic effects, while in the poloidal direction by the antenna pattern as mentioned in 2.1.1.

\subsection{Applications}

The main field of application of the CECE is the study of microinstabilities and turbulence in tokamak plasmas, one of the causes of anomalous transport. The very narrow IF bandwidth of the YIG filters combined with correlation analysis allows the study of extremely low scale perturbations; the high filtering frequency in the video stage was chosen to properly take into account the effects of high
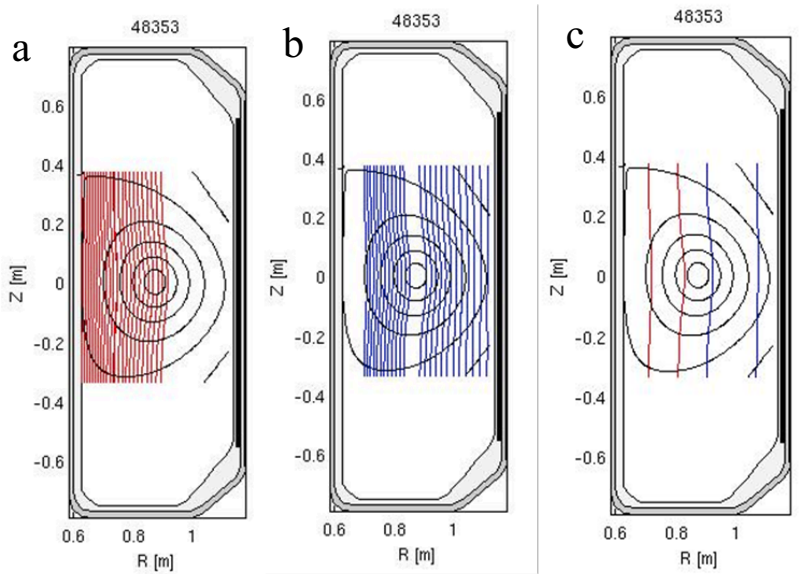

Figure 2. Representation of the resonance layers for the channels of (a) HFS ECE, (b) LFS ECE and (c) the two bands that can be accessed with the tunable YIG filters using the two different RF branches of the CECE.

frequency broadband turbulence. Finally the possibility to select the plasma volumes to analyse (by changing the center frequency of the YIG filters) over a wide radial range adds a further parameter that can be varied between similar shots to reconstruct profiles of turbulence characteristics.

In 2013 a series of experiments was performed with the goal of studying the effect of plasma shaping, in particular of the triangularity $\delta$, on turbulence characteristics in discharges with constant density and collisionality. It was found that negative $\delta$ leads to a narrower turbulence frequency range and larger correlation length and that these effects tend to be more evident moving outward from the plasma core, since that is the region where the $\delta$ differ- 

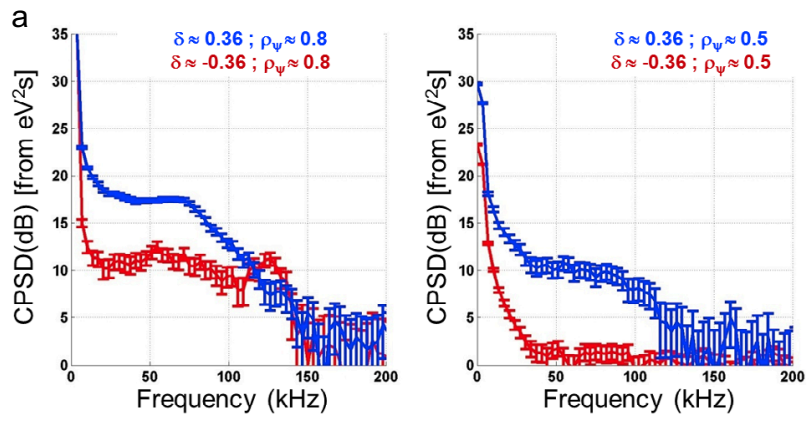

b

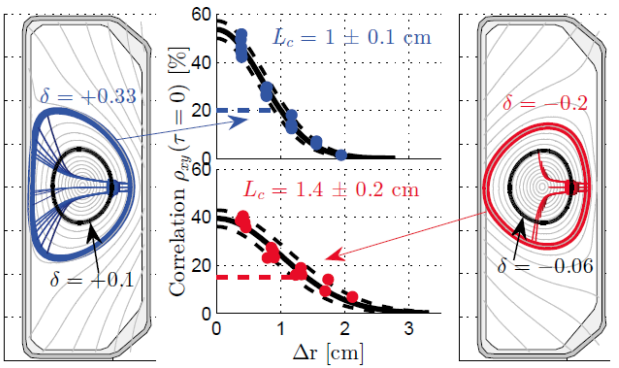

Figure 3. (a) Cross correlation spectra for two CECE channels at different radial positions $(\rho)$ in shots with positive and negative $\delta$ (b) Correlation length values for shots with positive and negative $\delta$.

ence is bigger [6], as shown in figure 3 . Given the upgrade of the CECE system, it is planned to expand the study of turbulence characteristics in a variety of operating conditions, including variations of density, shape, heating power, $T_{e} / T_{i}$ (exploiting the recently installed $1 \mathrm{MW}$ Neutral Beam Heating system), and regimes characterized by the presence of internal transport barriers. The results of these experiments will be compared with gyrokinetic simulations through the use of a novel CECE synthetic diagnostic.

Together with other diagnostics CECE has been used to characterize Geodesic Acoustic Modes (GAM) in TCV. These modes have been observed for $\rho_{\psi}>0.7$ and its frequency matches the observation of other diagnostics such as magnetic coils or Tangential Phase Contrast Imaging (TPCI) [6], performed in different poloidal and toroidal positions

\section{Conclusions}

The ECE diagnostic is of fundamental importance to the study of plasma physics in magnetic confinement devices allowing the possibility of obtaining information on local electron temperature with very high spatial and temporal resolution. TCV is equipped with a very flexible system that is able to cover the whole plasma cross section looking from the HFS and from the LFS. An important asset of this system is the presence of two non conventional lines of sight, one looking at the plasma along a vertical direction, while the other is connected to an antenna steerable around two axes, one of which can be scanned during shots. TCV is also equipped with a CECE system that can be used to study microfluctuations and turbulence characteristics at very low spatial scale by means of spectral decorrelation analysis. Future work will take advantage of the improved capabilities of the CECE diagnostic to study electron temperature fluctuations in a broad range of operating conditions.

\section{Acknowledgements}

This project has received funding from the HGF Virtual Institute on Plasma Dynamical Processes and Turbulence Studies using Advanced Microwave Diagnostics. This work was also supported in part by the Swiss National Science Foundation.

\section{References}

[1] T.C. Luce, P.C. Efthimion, N.J. Fisch, Review of Scientific Instruments 59, 1593 (1988)

[2] W.W. Eshetua, L. Porte, A. Fasoli, O. Sauter, S. Coda, T.P. Goodman, The TCV Team, EPJ Web of Conferences 32, 03011 (2012)

[3] T.P. Goodman, V.S. Udintsev, I. Klimanov, A. Mueck, O. Sauter, C. Schlatter, FST 53, 196 (2008)

[4] I. Klimanov, L. Porte, S. Alberti, P. Blanchard, A. Fasoli, T.P. Goodman, Review of Scientific Instruments 76, 093504 (2005)

[5] C. Watts, FST 52, 176 (2007)

[6] L. Porte et al., in Proceedings of 25th IAEA Fusion Energy Conference, St Petersburg, Russia (2014) 